\title{
Effect of older age at initiation of antiretroviral therapy on patient retention in an urban ART program in Uganda
}

This article was published in the following Dove Press journal:

Neurobehavioral HIV Medicine

I5 December 2010

Number of times this article has been viewed

Etheldreda

Nakimuli-Mpungu',2

Noeline Nakasujja ${ }^{2}$

Howard Dickens Akena ${ }^{2}$

Steven Mpungu Kiwuwa ${ }^{3}$

Elly Katabira ${ }^{4}$

Elilialia Okello

Chiadi Onyike ${ }^{5}$

Seggane Musisi²

'Mental Health Department, Johns Hopkins Bloomberg School of Public Health, Baltimore, MD, USA;

${ }^{2}$ Departments of Psychiatry, ${ }^{3}$ Clinical Epidemiology and Biostatistics,

${ }^{4}$ Internal Medicine, Makerere College of Health Sciences, School of Medicine, Kampala, Uganda; ${ }^{5}$ Division of Geriatric Psychiatry and Neuropsychiatry, Johns Hopkins Hospital, Baltimore, MD, USA
Correspondence: Etheldreda Nakimuli-Mpungu

Mental Health Department, Johns

Hopkins Bloomberg School of Public

Health, 624 N Broadway, Hampton

House, Baltimore, MD 21205 , USA

$\mathrm{Tel}+\mathrm{I} 4438394012$

Fax + I 4109559088

Email enakimul@jhsph.edu
Purpose: Patient retention in antiretroviral therapy (ART) programs in Sub-Saharan Africa is estimated at $60 \%$. There is limited information on the effect of older age ( $>50$ years) at ART initiation on patient retention in ART programs in Uganda. This study aimed to investigate demographic and clinical differences between older and younger human immunodeficiency virus (HIV)-positive individuals prior to ART initiation and then to assess the effect of older age at ART initiation on patient retention in an ART program.

Methods: A retrospective chart review of all medical charts belonging to 773 HIV-positive individuals who initiated ART from January 2005 through July 2009 was conducted. Factors associated with older HIV-positive individuals were determined using simple and multivariate logistic regression. Survival analysis techniques were used to compare median survival times in the ART program between older and younger HIV-positive individuals. Cox regression models were used to assess the impact of older age on patient retention while adjusting for variables associated with both older age and patient retention.

Results: Older HIV-positive individuals were significantly more likely to have a clinical diagnosis of HIV-related dementia (odds ratio [OR] $=7.65 ; P=0.037$ ), severe mental illness $(\mathrm{OR}=1.28 ; P=0.39)$, peripheral neuropathy, $(\mathrm{OR}=3.76 ; P=0.024)$, and HIV wasting syndrome $(\mathrm{OR}=1.92 ; P=0.023)$. Median survival time for older HIV-positive individuals was eight months longer than that of their younger counterparts $\left(\log\right.$-rank $\left.\chi^{2}=3.3, P=0.45\right)$ Independent predictors of loss to follow-up were a clinical diagnosis of HIV-related dementia (hazards ratio $[\mathrm{HR}]=4.63 ; P=0.000)$, severe mental illness $(\mathrm{HR}=1.44 ; P=0.005)$, and $\mathrm{HIV}$ wasting syndrome $(\mathrm{OR}=1.35 ; P=0.008)$.

Conclusion: There was no difference in patient retention rates between younger and older HIV-positive individuals. However, a clinical diagnosis of HIV dementia, severe mental disorder, HIV wasting syndrome, and CD4 counts less than 200 were independent predictors of low patient retention rates in this ART program.

Keywords: old age, human immunodeficiency virus, acquired immune deficiency syndrome, antiretroviral therapy, neuropsychiatric syndromes, Uganda

\section{Introduction}

In Uganda, massive efforts to scale up the provision of antiretroviral therapy (ART) to all regional and district government hospitals have led to the establishment of more ART programs countrywide. ${ }^{1,2}$ Although the main focus is to provide ART to all who need it, we are faced with the challenge of ensuring that those who receive ART take the treatments as prescribed, in order to prevent the emergence of drug-resistant strains of human immunodeficiency virus (HIV). ${ }^{3,4}$ Long-term retention of patients in Uganda's rapidly expanding ART programs enables us to investigate and gain 
insight into the biologic, social, and psychologic challenges faced by HIV-positive individuals receiving ART. A recent systematic review of patient retention in ART programs in Sub-Saharan Africa reported that ART programs in Africa retained about $60 \%$ of their patients at the end of two years of treatment. ${ }^{5}$ The authors concluded that more research is needed to better understand the reasons for attrition from ART programs.

This paper focuses on the effect of older age at ART initiation on patient retention in an urban ART program in Uganda. Few studies have directly examined how age impacts patient retention in ART programs in Sub-Saharan Africa. A recent HIV serobehavioral survey conducted by the Ministry of Health in Uganda revealed that the age-specific HIV prevalence rate among individuals in the 50-59 year age category, estimated at $11.5 \%$, was almost double the age-specific prevalence rate among individuals in the 15-24 year age category, estimated at $6.2 \% .{ }^{6}$ High prevalence rates of HIV infection in older individuals have also been reported in other African countries. The Swaziland Demographic and Health Survey conducted in 2006-2007 revealed an unusually high HIV prevalence among older age groups, with about $28 \%$ of men and $24 \%$ of women aged 50-54 years found to be HIV-positive. ${ }^{7}$

The epidemiology of HIV infection among adults older than 50 years is unknown in most Sub-Saharan African countries. Thus, our knowledge of factors associated with increasing numbers of older HIV-positive individuals in our ART programs is limited. This is problematic because both HIV infection and aging are important predictors of neurobehavioral syndromes. ${ }^{8,9}$

One ART program in Uganda has evaluated a sample of HIV-infected adults older than 60 years. The authors report substantial rates of behavioral risk factors for transmission of HIV, such as having multiple sexual partners and low rates of condom use in this sample, but no comparisons were made with younger HIV-positive individuals. ${ }^{10}$ Previous studies in the developed countries have attributed increasing numbers of older HIV-positive populations to changes in behavior that have resulted in acquisition of HIV infection at an older age $^{11,12}$ and a lack of clinical suspicion of HIV infection, which leads to delayed diagnosis in older individuals. ${ }^{13,14}$

In addition to these social issues, biologic and psychologic changes that are associated with aging may pose unique challenges to older HIV-positive individuals. ${ }^{15-18}$ In view of these facts, we hypothesize that older and younger HIV-positive individuals have different demographic and clinical characteristics. Consequently, retention of older HIV-positive individuals in ART programs would be lower than that of younger HIV-positive individuals. The goal of this study was to provide information that may add important insight and depth to our current understanding of how older age or factors associated with older age may impact patient retention in ART programs in Uganda.

\section{Methods}

\section{Study design, setting, and population}

This retrospective study was conducted at Butabika Hospital, a government-owned national referral mental hospital located on the shores of Lake Victoria in Kampala district. Data were collected as part of an ongoing retrospective medical chart review investigating patient characteristics and clinical outcomes of individuals receiving care at the Butabika Hospital outpatient center. This outpatient center houses a mental health clinic and general medical clinics, which service the surrounding urban community. Among the medical clinics is an HIV clinic, which was established in 2004.

The hospital started its ART program in 2005 and receives support from the Ugandan Ministry of Health, which is supported by the Global Fund to Fight AIDS, Tuberculosis and Malaria. Since 2005, this ART program has enrolled HIV-positive individuals from the surrounding urban community, as well as those discharged from the mental hospital after treatment of an episode of severe mental illness. By the end of 2009, this ART program had initiated 800 individuals on ART, of whom $25 \%$ had been treated for an episode of severe mental illness prior to ART initiation.

In this ART program, HIV-positive individuals are initiated on ART if they have a CD4 T cell count of 200 or less. In the absence of CD4 T cell counts, individuals with World Health Organization (WHO) clinical stage 3 or 4 disease and/or total lymphocyte count $<1500$ cells $/ \mathrm{mm}^{3}$ are considered eligible for ART. First-line ART is a fixed-dose combination of stavudine, lamivudine, and nevirapine. All individuals receive baseline clinical evaluations prior to ART initiation in which information on demographic and clinical information, including HIV symptoms, CD4 counts (whenever possible), acquired immune deficiency syndrome (AIDS) diagnosis, and psychiatric diagnosis, is obtained. The ART program runs a clinic every Wednesday where new and continuing patients receive clinical assessments depending on their presenting health problems. Individuals initiated on ART are given medications to last for one month and are required to make monthly clinic visits for assessment and medication refills. 


\section{Data collection}

Trained research assistants reviewed all medical charts of individuals who initiated ART from January 2005 to July 2009 and extracted data on variables of interest. Among the demographic data extracted were age at ART initiation, gender, marital status (single, married, widowed, divorced/ separated), and employment status (employed versus unemployed). Among clinical characteristics, data were extracted on number of HIV-related symptoms/opportunistic infections, baseline CD4 counts, and the presence of a psychiatric diagnosis. The date of ART initiation and the date of the last clinic visit were extracted. Individuals whose last visit was more than three months prior to the date of administrative censoring were considered to have dropped out of the program. In this study, the exposure of interest was age (older age $\geq 50$ years versus younger age $<50$ years) at ART initiation. The outcome of interest was time spent in the ART program since ART initiation. The "failure event" was dropping out of the ART program. Individuals whose last visit was more than three months prior to the date of administrative censoring were considered to have dropped out of the program. The study was approved by the Butabika Hospital Research Ethics Committee.

\section{Statistical analysis}

Statistical analysis was performed using Stata 10.0 (Stata Corp, College Station, TX, USA). Simple and multiple logistic regressions were conducted to identify demographic and clinical variables that were significantly correlated with older age at initiation of ART. We computed Kaplan-Meier estimates and log-rank Chi-square tests to determine the impact of study variables on patient retention in the ART program. Study variables that were statistically significantly associated with older age and patient retention were entered into a Cox regression model to control for their effect when assessing the effect of older age on patient retention in the ART program. We used graphic methods and Schoenfeld residuals to assess the proportional hazards assumption. ${ }^{19}$

\section{Results \\ Subjects}

Data were abstracted from 773 medical charts of HIV-positive individuals, who initiated ART from January 2005 to July 2009. Over this 4.5 -year period, the dropout rate was 37 per 100 person-years, with a total of $334 \mathrm{HIV}$-positive individuals having dropped out of the ART program by July 30, 2009. At the time of administrative censoring, median survival time in this ART program was estimated at 2.5 years. Among those lost to follow-up, death was ascertained in only 12 individuals from family and friends. For individuals who transferred to other programs, we assumed that they had continued with their HIV medications in another ART program, and thus they were considered as having been retained in care. Overall, study participants had a mean age of 35.5 years (standard deviation $[\mathrm{SD}]=9.2$ ), age range (11-82 years), and male to female ratio 1:2.

\section{Older versus younger HIV positivity}

The numbers of older HIV-positive individuals who initiated ART each year (from January 2005 to July 2009), respectively, were 7, 26, 14, 27, and 12. They accounted for $11.13 \%$ of all HIV-positive individuals who have initiated ART in this program. They had a mean age of $54.8(\mathrm{SD}=6.53)$, age range 50-82 years, and male to female ratio 1:1.2.

Younger HIV-positive individuals accounted for $89 \%$ of all individuals in the ART program. Their mean age was 34 years $(\mathrm{SD}=7.65)$, age range $11-49$ years, and male to female ratio 1:2. Details of differences in baseline demographic and clinical characteristics of older and younger HIV-positive individuals prior to ART initiation are shown in Table 1. Details of differences in baseline HIV-related symptoms reported by older and younger HIV-positive individuals are shown in Table 2. Factors independently associated with older HIV-positive individuals after multivariate analysis are shown in Table 3.

\section{Effects of variables associated with older HIV-positive individuals on retention}

Figure 1 shows the Kaplan-Meier estimates of the distribution of time since ART initiation to attrition from the ART program according to age. The median survival time was 2.1 years for younger HIV-positive individuals and 2.75 years for older HIV-positive individuals. This means that at both these time points, the proportion of older HIV-positive individuals in the ART program was higher than that of younger HIVpositive individuals. However, this difference did not attain statistical significance (log-rank test $\chi^{2}=1.4, P=0.45$ ).

Individuals with HIV wasting syndrome, those with a severe mental disorder, and those who were clinically diagnosed with HIV-related dementia were more likely to drop out of the ART program compared with individuals without these conditions (log-rank test $\chi^{2}=13.2, P<0.001$; log-rank test $\chi^{2}=20.8, P<0.001 ;$ log-rank test $\chi^{2}=22.4$, $P<0.001)$, respectively. No significant gender differences 
Table I A comparison of demographic and clinical characteristics of older ( $\geq 50$ years) and younger ( $<50$ years) HIV-positive individuals prior to ART initiation in Uganda

\begin{tabular}{|c|c|c|c|c|}
\hline Characteristic & $\begin{array}{l}\geq 50 \text { years HIV- } \\
\text { positive }(n=86) \%\end{array}$ & $\begin{array}{l}<50 \text { years HIV- } \\
\text { positive }(n=687) \%\end{array}$ & $\begin{array}{l}\text { Unadjusted } \\
\text { odds ratio }\end{array}$ & $P$ value \\
\hline \multicolumn{5}{|l|}{ Gender } \\
\hline Male & 43 & 34.1 & 1 & \\
\hline Female & 53.4 & 64.6 & 0.65 & 0.07 \\
\hline Gender missing & 3.5 & 1.31 & 2.10 & 0.28 \\
\hline \multicolumn{5}{|l|}{ Marital status } \\
\hline Single & 11.6 & 21.3 & 1 & \\
\hline Married & 27.9 & 31.2 & 1.64 & 0.20 \\
\hline Separated/divorced & 2.3 & 3.2 & 1.32 & 0.73 \\
\hline Widow & 12.8 & 7.6 & 3.08 & 0.015 \\
\hline Marital status missing & 45.4 & 36.6 & 2.25 & 0.028 \\
\hline \multicolumn{5}{|l|}{ Employment status } \\
\hline Unemployed & 22.1 & 16 & 1 & \\
\hline Employed & 31.4 & 41.1 & 0.55 & 0.06 \\
\hline Employment missing & 46.5 & 42.9 & 0.79 & 0.42 \\
\hline \multicolumn{5}{|l|}{ CD4 counts } \\
\hline CD4 $<200$ & 65.1 & 66.8 & I & \\
\hline$C D 4 \geq 200$ & 16.3 & 15.4 & I.I & 0.80 \\
\hline CD4 missing & 18.6 & 17.8 & I.I & 0.81 \\
\hline \multicolumn{5}{|c|}{ Opportunistic infection } \\
\hline No tuberculosis & 88.2 & 88.9 & I & \\
\hline Previous tuberculosis & 7.1 & 4.1 & 1.74 & 0.23 \\
\hline Current tuberculosis & 4.7 & 7 & 0.67 & 0.47 \\
\hline \multicolumn{5}{|c|}{ Any severe mental disorder } \\
\hline Absent & 65.9 & 76.9 & $\mathrm{I}$ & \\
\hline Present & 34.1 & 23.1 & 1.72 & 0.027 \\
\hline \multicolumn{5}{|c|}{ Clinical diagnosis of HIV dementia } \\
\hline Absent & 96.5 & 99.6 & I & \\
\hline Present & 3.5 & 0.4 & 9.8 & 0.006 \\
\hline \multicolumn{5}{|l|}{ Toxoplasmosis } \\
\hline Absent & 98.8 & 95.9 & I & \\
\hline Present & 1.2 & 4.1 & 0.33 & 0.28 \\
\hline \multicolumn{5}{|l|}{ Kaposi sarcoma } \\
\hline Absent & 98.8 & 98.5 & 1 & \\
\hline Present & 1.2 & 1.5 & 0.94 & 0.95 \\
\hline
\end{tabular}

Abbreviations: ART, antiretroviral therapy; HIV, human immunodeficiency virus.

were observed in patient retention (log-rank test $\chi^{22}=1.04$, $P<0.59$ ). Table 4 shows the Cox proportional hazard models for covariates with rate of retention since ART initiation to attrition from the ART program. After controlling for study variables associated with both older age and rate of retention in the ART program, older age did not emerge as an independent predictor of patient retention. However, neuropsychiatric conditions independently associated with older age emerged as predictors of patient retention in the ART program.

\section{Discussion}

This study has three major findings. First, in keeping with our study hypothesis, there were some demographic differences between older and younger HIV-positive individuals. Individuals 50 years and older accounted for $11 \%$ of the total population who initiated ART over a 4.5-year period. Of these, $5 \%$ were aged 65 years and older. Furthermore, they were less likely to be female and more likely to have lost a spouse. These findings are similar to findings from the US HIV Cost and Service Utilization Study (HCSUS) in which $11 \%$ of 2864 HIV-positive individuals at baseline were more than 50 years old and $5 \%$ were aged 65 years and older. ${ }^{20}$ Similar to our study population, the older HCSUS population comprised fewer females than males. Another study that evaluated older HIV-positive individuals in the Congo also reported fewer females than males in their study sample. ${ }^{16}$ The preponderance of males who had lost their spouses 
Table 2 HIV-related symptoms reported by older ( $>50$ years) and younger ( $<50$ years) HIV-positive individuals prior to ART initiation in Uganda

\begin{tabular}{|c|c|c|c|c|}
\hline Characteristic & $\begin{array}{l}\geq 50 \text { years HIV- } \\
\text { positive }(n=86) \%\end{array}$ & $\begin{array}{l}<50 \text { years HIV- } \\
\text { positive }(n=687) \%\end{array}$ & $\begin{array}{l}\text { Unadjusted } \\
\text { odds ratio }\end{array}$ & $P$ value \\
\hline \multicolumn{5}{|c|}{ HIV wasting syndrome } \\
\hline Absent & 44.2 & 60.6 & I & \\
\hline Present & 55.8 & 39.4 & 1.99 & 0.005 \\
\hline \multicolumn{5}{|c|}{ Recurrent fevers } \\
\hline Absent & 68.6 & 58.7 & I & \\
\hline Present & 31.4 & 41.3 & 0.65 & 0.07 \\
\hline \multicolumn{5}{|c|}{ Respiratory tract infections } \\
\hline Absent & 63.9 & 58.5 & 1 & \\
\hline Present & 36.1 & 41.5 & 0.79 & 0.33 \\
\hline \multicolumn{5}{|c|}{ Recurrent diarrhea } \\
\hline Absent & 86.1 & 86.8 & I & \\
\hline Present & 13.9 & 13.2 & I.I & 0.85 \\
\hline \multicolumn{5}{|l|}{ Oral candidiasis } \\
\hline Absent & 83.7 & 84.7 & I & \\
\hline Present & 16.3 & 15.3 & 1.10 & 0.81 \\
\hline \multicolumn{5}{|c|}{ Genital/rectal infections } \\
\hline Absent & 96.5 & 96.5 & I & \\
\hline Present & 3.5 & 3.5 & 0.99 & 0.99 \\
\hline \multicolumn{5}{|l|}{ Skin rashes } \\
\hline Absent & 83.7 & 79.5 & 1 & \\
\hline Present & 16.3 & 20.5 & 0.75 & 0.36 \\
\hline \multicolumn{5}{|c|}{ Peripheral neuropathy } \\
\hline Absent & 91.9 & 96.7 & 1 & \\
\hline Present & 8.14 & 3.3 & 3.07 & 0.013 \\
\hline \multicolumn{5}{|l|}{ Dizziness } \\
\hline Absent & 95.4 & 95.8 & I & \\
\hline Present & 4.6 & 4.4 & I.II & 0.85 \\
\hline
\end{tabular}

Abbreviations: ART, antiretroviral therapy; HIV, human immunodeficiency virus.

in our sample of older HIV-positive individuals probably reflects the nature of the epidemic in most Sub-Saharan African countries, where females are usually infected with HIV infection at a much younger age compared with males. ${ }^{21}$ Thus, the majority of HIV-infected females may not survive to the age of 50 years.

Second, there were some clinical differences between older and younger HIV-positive individuals. Our study confirms the previous finding from developed countries ${ }^{22,23}$ that older HIV-positive individuals are more likely to have neuropsychiatric conditions, including severe mental disorders, clinically diagnosed HIV-related dementia, and peripheral neuropathy compared with their younger counterparts. Prevalence estimates of these neuropsychiatric conditions among older HIV-infected individuals in our study were $34.12 \%$, $3.5 \%$, and $8.14 \%$, respectively. Among younger HIV-infected individuals, prevalence estimates of these neuropsychiatric conditions were $23.1 \%, 0.4 \%$, and $3.3 \%$, respectively. Contrary to findings from developed countries, ${ }^{15,24}$ these neuropsychiatric conditions were not associated with advanced HIV disease. Both groups had comparable baseline CD4 counts, and there were no significant associations between

Table 3 Factors independently associated with older age among HIV-positive individuals prior to ART initiation

\begin{tabular}{llll}
\hline Baseline characteristics & Adjusted odds ratio & $\mathbf{9 5 \%} \mathbf{C l}$ & P value \\
\hline Female gender & 0.62 & $0.37-1.03$ & 0.06 \\
Loss of a spouse & 3.23 & $1.20-8.76$ & 0.02 \\
Clinical diagnosis of HIV dementia & 7.65 & $1.13-51.6$ & 0.037 \\
Peripheral neuropathy & 3.76 & $1.08-7.22$ & 0.024 \\
HIV wasting syndrome & 1.92 & $1.17-3.15$ & 0.009 \\
Severe mental disorder & 1.28 & $0.69-2.16$ & 0.39 \\
\hline
\end{tabular}

Abbreviations: ART, antiretroviral therapy; $\mathrm{Cl}$, confidence interval; $\mathrm{HIV}$, human immunodeficiency virus. 


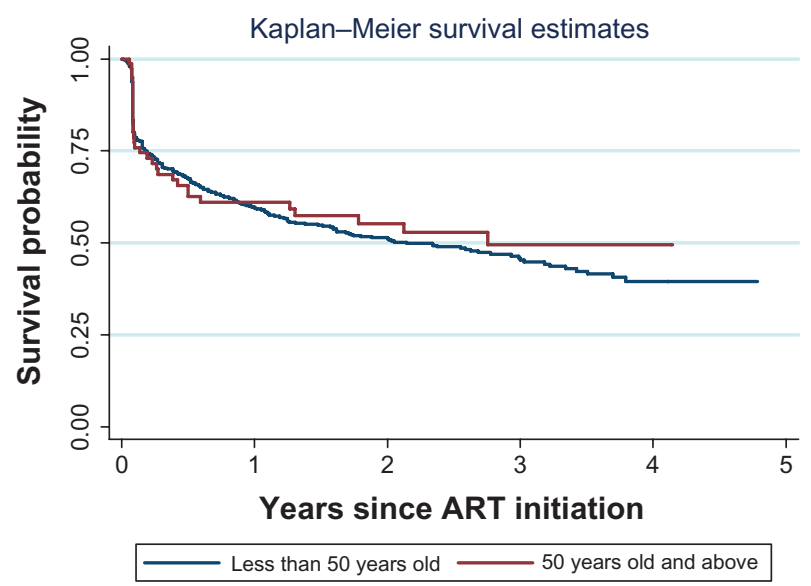

Figure I Kaplan-Meier estimate of the distribution of time from ART initiation until attrition from the ART program according to age.

Abbreviation: ART, antiretroviral therapy.

the specific neuropsychiatric conditions and CD4 counts. This finding supports those of previous studies performed in Uganda, which found that neuropsychiatric conditions in HIV-positive individuals do not predominantly occur in the advanced stage of HIV disease but can occur both in the early and late stages of the disease. ${ }^{25-28}$

Another clinical difference was that older HIV-positive individuals were more likely to receive a diagnosis of HIV wasting syndrome despite having lower or comparable rates of symptoms related to infectious diseases, such as oral candidiasis, recurrent fevers, diarrhea, respiratory infections, and genital infections compared with their younger counterparts. This finding is similar to that from a study in the Congo that evaluated clinical characteristics of HIV-positive individuals aged 55 years and older. ${ }^{16}$ All study participants were diagnosed with HIV wasting syndrome, but no comparisons were made with younger HIV-positive individuals. High rates of HIV wasting syndrome in older HIV-positive individuals may be explained by the synergistic effects of HIV infection and aging on body composition. Studies have shown that muscle mass may decline significantly because of the aging process $^{29}$ or HIV infection. ${ }^{30}$

Table 4 Independent predictors of attrition from ART program

\begin{tabular}{llll}
\hline Baseline characteristics & Hazard ratio & $\mathbf{9 5 \%} \mathbf{C I}$ & $\boldsymbol{P}$ value \\
\hline Being $\geq 50$ years old & 0.85 & $0.59-1.23$ & $0.4 \mathrm{I}$ \\
$\begin{array}{l}\text { Clinical diagnosis of HIV } \\
\text { dementia }\end{array}$ & 4.63 & $2.65-8.35$ & $<0.00 \mathrm{I}$ \\
$\begin{array}{l}\text { Peripheral neuropathy } \\
\text { HIV wasting syndrome }\end{array}$ & 0.54 & & \\
Severe mental disorder & 1.35 & $0.28-1.04$ & 0.06 \\
CD4 count $>200$ & 1.44 & $1.08-1.68$ & 0.008 \\
\hline
\end{tabular}

Abbreviations: ART, antiretroviral therapy; $\mathrm{Cl}$, confidence interval; HIV, human immunodeficiency virus.
Third, contrary to our hypothesis, patient retention rates in the Butabika Hospital ART program appeared to be higher among older than younger HIV-positive individuals. However, the association was weak and did not attain statistical significance. There are conflicting reports about differences in clinical outcomes between older and younger HIV-infected persons. Some studies have linked faster progression to AIDS and shorter survival rates with older age among HIV-positive individuals. ${ }^{31-33}$ Other studies that have investigated predictors of ART outcome have found that older HIV-positive individuals adhere better to antiretroviral medications and have better virologic outcomes than younger ones. ${ }^{34,35}$

One reason for the conflicting results could be that older individuals with rapidly progressing HIV disease may not make it into the ART programs, and, if they do, they may have poor outcomes. Older individuals with mild, slowly progressing HIV disease are more likely to survive long enough to initiate ART and thus may have better treatment outcomes. Although older age did not seem to affect patient retention in this ART program significantly, neuropsychiatric conditions associated with older age were independent predictors of low patient retention rates in our ART program.

We must be cautious in interpreting the data from this study. First, the retrospective chart review nature of our study confers a number of limitations. The accuracy of data extracted from chart reviews relies in turn on the accuracy of written records and/or recall of individuals. Thus, individuals with more advanced disease may not be able to report all their symptoms as a result of their present physical state or cognitive impairment. Second, important data were not available in this ART program, eg, prospectively collected information on education status, metabolic complications of HIV infection, ART adherence, and immunologic and virologic response. These missing variables may be potential confounders of the association between older age and patient retention in the ART program. Failure to adjust for these variables may lead to biased estimates of the measures of association. Third, previous research has shown that psychiatric dysfunction may precede infection with HIV or develop as a direct or indirect consequence of the disease. Given that we do not have data on past psychiatric illness or family history of psychiatric disorders, we cannot attribute neuropsychiatric disorders in older HIV-positive individuals to HIV infection alone. Furthermore, we do not have data on numerous factors that could lead to psychiatric disorders in the elderly, such as increased rates of neurologic disease and neurotransmitter abnormalities, adverse reaction to medications (prescribed or otherwise), physical disability, social 
isolation, and bereavement. Thus, we cannot ascribe their neuropsychiatric conditions to any specific etiologic factor. Fourth, some variables had missing data. Except for marital status, other missing variables, ie, gender, employment, and CD4 counts, were not associated with either the exposure or outcome of interest. Nevertheless, we handled the missing data using the missing indicator method. ${ }^{36}$ This method does not exclude subjects from the analysis but adds an extra variable to the statistical model to indicate that the value of a certain variable is missing. Using this method, missing values are consequently adjusted for in the statistical model, so there is minimal residual confounding. Fifth, it should be noted that the ideal cutoff point for defining older individuals is 65 years. However, we adopted the cutoff of 50 years based on recommendations from the National Institutes of Health. Lastly, it is difficult to establish cause and effect from a retrospective chart review. Given the limited resources in a poor country like Uganda, where prospective studies may not be readily feasible, the results of this retrospective study are, at best, hypothesis-generating and may identify feasibility issues for a prospective study.

\section{Clinical implications}

Within the context of the aforementioned limitations, this study provides information on prevalence rates of severe mental disorders and clinically diagnosed dementia in relation to prevalence estimates of other important HIV-related conditions, such as tuberculosis, in a sample of older HIV-positive individuals. It is evident that the burden of neuropsychiatric conditions in older HIV-positive individuals is higher than that of other infectious diseases. Caution must be exercised when prescribing ART, making sure that older HIV-positive individuals are not exposed to ART regimens with adverse effects including neuropsychiatric symptoms.

The unique comorbidity of neuropsychiatric disorders and HIV/AIDS among older individuals presents unique challenges to HIV care providers. Although guidelines for ART are described for children, adolescents, ${ }^{37}$ and adults, ${ }^{38}$ there are no specific guidelines for older patients with HIV infection. Previous research has shown that older age may potentiate the deleterious neurocognitive effects of HIV infection. ${ }^{39}$ Given the relative dearth of knowledge regarding the epidemiology of neurocognitive and psychiatric disorders in older HIV-positive adults in Uganda, basic epidemiologic research into the incidence and prevalence of HIV-associated cognitive and psychiatric disorders in the elderly is of paramount importance. In addition, research is needed to determine whether the nature, severity, course, and treatment of HIV-associated neuropsychiatric and neurocognitive disease differs between older HIV-positive adults and younger HIV-positive comparison groups, as well as older uninfected controls. This research would provide information on whether there is simply an additive or a true synergistic effect of both advancing age and HIV infection.

\section{Conclusion}

In this ART program, there was no difference in patient retention rates between younger and older HIV-positive individuals. However, a clinical diagnosis of HIV dementia, severe mental disorder, or HIV wasting syndrome and CD4 counts less than 200 were independent predictors of low patient retention rates in this ART program. More research studies using better epidemiologic designs are needed to confirm these findings.

\section{Disclosure}

The authors report no conflicts of interest in this work.

\section{References}

1. Amolo-Okero F, Aceng E, Madraa M, Namagala E. Scaling up antiretroviral therapy: Experience in Uganda case study. Available at: http:// www.who.int/hiv/amds/case3.pdf. Accessed 2010 Nov 16.

2. The National Strategic Framework for Expansion of HIV/AIDS Care and Support in Uganda, 2001/2-2005/6. Kampala; Ministry of Health: 2002 Feb. Available at: http://www.aidsuganda.org/mtr/ Annex_2_TWG_2_Report.pdf. Accessed 2010 Nov 16.

3. Bangsberg DR. Preventing HIV antiretroviral resistance through better monitoring of treatment adherence. J Infect Dis. 2008;197: S272-S278.

4. Gardner EM, Burman WJ, Steiner JF, Anderson PL, Bangsberg DR Antiretroviral medication adherence and the development of classspecific antiretroviral resistance. AIDS. 2009;23:1035-1046.

5. Rosen S, Fox MP, Gill CJ. Patient retention in antiretroviral therapy programs in sub-Saharan Africa: A systematic review. PLoS Med. 2007;4:e298.

6. Uganda Ministryof Health (2006) 2004-05 Uganda HIV/AIDS serobehavioural survey. Available at: http://www.measuredhs.com/pubs/ pdf/AIS2/AIS2.pdf. Accessed 2010 Nov 16.

7. The Swaziland Demographic and Health Survey (2006-2007). Available at: http://www.safaids.net/files/Swaziland\%20Demographic\%20 and\%20Health\%20Survey\%202006-2007.pdf. Accessed 2010 Nov 16.

8. Becker JT, Lopez OL, Dew MA, Aizenstein HJ. Prevalence of cognitive disorders differs as a function of age in HIV virus infection. AIDS. 2004;18:S11-S18.

9. Hinkin CH, Castellon SA, Atkinson JH, Goodkin K. Neuropsychiatric aspects of HIV infection among older adults. J Clin Epidemiol. 2001;54:S44-S52.

10. Nakasujja N, Musisi S, Byakika-Kibwika P, Katabira E. HIV/AIDS among the elderly: A sociodemographic survey at the Mildmay Clinic Kampala, Uganda. Proceedings of the 4th Annual Makerere University College of Health Sciences Scientific Conference. Kampala, Uganda, 2008 Sep 17-19.

11. Stoff DM. Mental health research in HIV/AIDS and aging: Problems and prospects. AIDS. 2004;18:S3-S10. 
12. Kooperman L. A survey of gay and bisexual men age 50 and older. AIDS related knowledge, attitude, belief, and behavior. AIDS Patient Care. 1994;8:114-117.

13. Stall R, Catania J. AIDS risk behaviors among late middle-aged and elderly Americans. The national AIDS behavioral surveys. Arch Intern Med. 1994;154:57-63.

14. Zablotsky D, Kennedy M. Risk factors and HIV transmission to midlife and older women: Knowledge, options, and the initiation of safer sexual practices. J Acquir Immune Defic Syndr. 2003;33:S122-S130.

15. Justice AC, McGinnis KA, Atkinson JH, et al; Veterans Aging Cohort 5-Site Study Project Team. Psychiatric and neurocognitive disorders among HIV-positive and negative veterans in care: Veterans aging cohort five-site study. AIDS. 2004;18:S49-S59.

16. Ibara JR, Itoua C, Gathse A, et al. Acquired immunodeficiency syndrome in elderly persons in a tropical zone. Apropos of 175 cases in the Congo. Bull Soc Pathol Exot. 2002;95:100-102.

17. Biggar RJ, Kirby KA, Atkinson J, McNeel TS, Engels E; AIDS Cancer Match Study Group. Cancer risk in elderly persons with HIV/AIDS. J Acquir Immune Defic Syndr. 2004;36:861-868.

18. Heckman TG, Heckman BD, Kochman A, Sikkema KJ, Suhr J, Goodkin K. Psychological symptoms among persons 50 years of age and older living with HIV disease. Aging Ment Health. 2002;6:121-128.

19. Klein JP, Moeschberger ML. Survival Analysis: Techniques for Censored and Truncated Data. New York, NY: Springer-Verlag; 1992.

20. Zingmond DS, Kilbourne AM, Justice AC, et al. Differences in symptom expression in older HIV-positive patients: The veterans aging cohort 3 site study and HIV cost and service utilization study experience. J Acquir Immune Defic Syndr. 2003;33:S84-S92.

21. Cohen CR, Montandon M, Carrico AW, et al. Association of attitudes and beliefs towards antiretroviral therapy with HIV-seroprevalence in the general population of Kisumu, Kenya. PLoS One. 2009;4:e4573.

22. Valcour V, Shikuma C, Shiramizu B, et al. Higher frequency of dementia in older HIV-1 individuals: The Hawaii aging with HIV-1 cohort. Neurology. 2004;63:822-827.

23. Baldeweg T, Catalan J, Gazzard BG. Risk of HIV dementia and opportunistic brain disease in AIDS and zidovudine therapy. J Neurol Neurosurg Psychiatry. 1998;65:34-41.

24. Goodkin K, Wilkie FL, Concha M, et al. Aging and neuro-AIDS conditions and the changing spectrum of HIV-1-associated morbidity and mortality. J Clin Epidemiol. 2001;54:S35-S43.

25. Akena DH, Musisi S, Kinyanda E. A comparison of the clinical features of depression in HIV-positive and HIV-negative patients in Uganda. Afr J Psychiatry (Johannesbg). 2010;13:43-51.

26. Nakimuli-Mpungu E, Musisi S, Mpungu SK, Katabira E. Primary mania versus HIV-related secondary mania in Uganda. Am J Psychiatry. 2006;163:1349-1354.
27. Nakimuli-Mpungu E, Musisi S, Kiwuwa Mpungu S, Katabira E. Early-onset versus late-onset HIV-related secondary mania in Uganda. Psychosomatics. 2008;49:530-534.

28. Nakimuli-Mpungu E, Musisi S, Mpungu SK, Katabira E. Clinical presentation of bipolar mania in HIV-positive patients in Uganda. Psychosomatics. 2009;50:325-330.

29. Kallman DA, Plato CC, Tobin JD. The role of muscle loss in the age-related decline of grip strength: Cross-sectional and longitudinal perspectives. J Gerontol. 1990;45:M82-M88.

30. Koethe JR, Heimburger DC. Nutritional aspects of HIV-associated wasting in sub-Saharan Africa. Am J Clin Nutr. 2010;91: S1138-S1142.

31. Operskalski EA, Mosley JW, Busch MP, Stram DO. Influences of age, viral load, and CD4+ count on the rate of progression of HIV-1 infection to AIDS. Transfusion safety study group. JAcquir Immune Defic Syndr Hum Retrovirol. 1997;15:243-244.

32. Soriano V, Castilla J, Gomez-Cano M, et al. The decline in CD4+ $\mathrm{T}$ lymphocytes as a function of the duration of HIV infection, age at seroconversion, and viral load. J Infect. 1998;36:307-311.

33. Adler WH, Baskar PV, Chrest FJ, Dorsey-Cooper B, Winchurch RA, Nagel JE. HIV infection and aging: Mechanisms to explain the accelerated rate of progression in the older patient. Mech Ageing Dev. 1997;96:137-155.

34. Hinkin CH, Hardy DJ, Mason KI, et al. Medication adherence in HIVinfected adults: Effect of patient age, cognitive status, and substance abuse. AIDS. 2004;18:S19-S25.

35. Nachega JB, Hislop M, Nguyen H, et al. Antiretroviral therapy adherence, virologic and immunologic outcomes in adolescents compared with adults in Southern Africa. J Acquir Immune Defic Syndr. 2009;51:65-71.

36. Knol MJ, Janssen KJ, Donders AR, et al. Unpredictable bias when using the missing indicator method or complete case analysis for missing confounder values: An empirical example. J Clin Epidemiol. 2010;63:728-736.

37. Working Group on Antiretroviral Therapy and Medical Management of HIV-infected Children convened by the National Pediatric and Family HIV Resource Center (NPHRC), the Health Resources and Services Administration (HRSA), and the National Institute of Health (NIH). Guidelines for the use of antiretroviral agents in pediatric HIV infection. MMWR. 2000:1-59.

38. Carpenter CCJ, Cooper DA, Fischl MA, et al. Antiretroviral therapy in adults: Updated recommendations of the International AIDS SocietyUSA Panel. JAMA. 2000;283:381-390.

39. Janssen RS, Nwanyanwu OC, Selik RM, Stehr-Green JK. Epidemiology of human immunodeficiency virus encephalopathy in the United States. Neurology. 1992;42:1472-1476.
Neurobehavioral HIV Medicine

\section{Publish your work in this journal}

Neurobehavioral HIV Medicine is an international, peer-reviewed, open access journal focusing on advances in research in HIV/ AIDS, with specific reference to the neurological, psychiatric and behavioral consequences of the disease, concomitant infections and specific antiretroviral therapy. The manuscript

\section{Dovepress}

management system is completely online and includes a very quick and fair peer-review system, which is all easy to use. Visit http://www.dovepress.com/testimonials.php to read real quotes from published authors. 